29th European Symposium on Computer-Aided Process

Engineering (ESCAPE-29), Eindhoven, Netherlands,

June 16-19, 2019, submitted

Anton A. Kiss, Edwin Zondervan, Richard Lakerveld, Leyla Özkan (Eds.)

Proceedings of the $29^{\text {th }}$ European Symposium on Computer Aided Process Engineering

June $16^{\text {th }}$ to $19^{\text {th }}$, 2019, Eindhoven, The Netherlands. () 2019 Elsevier B.V. All rights reserved.

\title{
Optimal Maintenance Scheduling for Washing of Com- pressors to Increase Operational Efficiency
}

Frederik Schulze Spüntrup ${ }^{\mathrm{a}}$, Giancarlo Dalle Ave ${ }^{\mathrm{b}, \mathrm{c}}$, Lars Imsland ${ }^{\mathrm{a},{ }^{*}}$ and Iiro Harjunkoski ${ }^{\mathrm{b}}$

${ }^{a}$ Department of Engineering Cybernetics, Norwegian University of Science and Technology, 7491 Trondheim, Norway

${ }^{\mathrm{b}}$ ABB Corporate Research Germany, 68526 Ladenburg, Germany

${ }^{\mathrm{c}}$ Technical University Dortmund, 7491 Dortmund, Germany

lars.imsland@ntnu.no

\begin{abstract}
The need for simultaneously decreasing carbon emissions and increasing operational profit motivates gas turbine and gas compressor operators to understand, minimize and control performance deterioration. Fouling is one of the most prevalent deterioration problems. This work addresses the causes and effects of fouling and investigates an approach to give decision-support on if, how often and when compressor washing should be conducted. Integration with other maintenance actions is also considered. In this work, a discrete time-scheduling approach that follows the Resource Task Network framework is developed and formulated as a Mixed Integer Linear Program. A novel enumerator formulation makes this method simpler and easier to extend for different maintenance types than existing methods. Degradation is included in a linearized way for a case study from the Oil and Gas industry. Results indicate that washing scheduling is beneficial for the profit. This study gives the foundation for decision-support regarding additional investments in existing production systems, amortization, and supplies optimal maintenance schedules for various applied maintenance types.
\end{abstract}

Keywords: Maintenance scheduling, asset degradation, energy efficiency

\section{Introduction and Background}

Degradation of assets is an omnipresent phenomenon in the process industries where the lifetime of a plant easily reaches 30-50 years. Deterioriation of assets is inevitable. Over the lifecycle of an equipment its efficiency will decrease and it will degrade up to a level where it cannot be operated any longer or the operating company decides to take countermeasures. According to Diakunchak (1992) compressor fouling constitutes $70-85 \%$ of the performance loss caused by deterioration in gas turbines. With the latest IPCC report on achieving the maximum $1.5^{\circ} \mathrm{C}$ average temperature increase goal it is important to avoid any unnecessary waste of energy.

While the literature identifies performance degradation and declares washing as an appropriate countermeasure (Stalder, 2001), there is not much work done on the decision when to perform the washing procedure. The contribution of this work is to include the efficiency degradation of assets into the decision on when to perform maintenance tasks. 


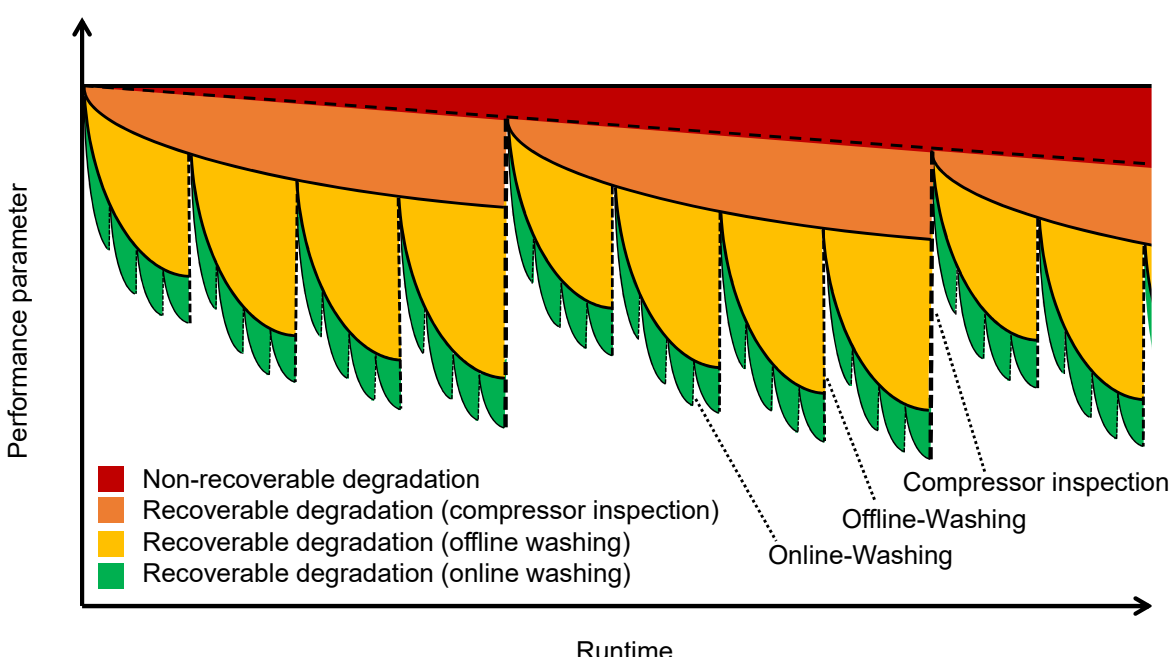

Figure 1: Example of the different types of degradation in a compressor and different types of maintenance to restore efficiency.

Kurz and Brun (2001) describe the degradation of gas turbine systems. Different mechanisms affect the degradation process: Increased tip clearance (Khalid et al., 1999) and changes in the airfoil geometry (Singh et al., 1996) are interlinked with the effect of nonrecoverable degradation. Recoverable degradation, which can be (partially) reversed by specific methods (e.g. washing) is described as changes in the airfoil surface quality (Elrod et al., 1990). Kurz and Brun (2012) reported significant deterioration of different gas turbines, ranging between 2 and $12 \%$ after one year of operation.

Hovland and Antoine (2006) optimise the power generation by applying parameter estimation via Kalman filtering combined with a hybrid dynamic model. Model predictive control is used with this hybrid dynamic model and compared to fixed washing schedules.

Xenos et al. (2016) investigate an air separation plant. Optimal operation and the maintenance of compressor networks are part of the developed MILP model. Online and offline washing are considered as part of the condition-based maintenance approach. The degradation is considered in three different cases: Low, medium and high degradation. They are based on a study of industrial data. Offline-washing is not considered to be done during non-performance-related maintenance actions or regular shutdowns of the equipment. The degradation in the case study causes an increase in electrical power consumption of $2 \%$ per month, which leads to high saving potential.

This paper addresses optimal scheduling of maintenance actions to increase operational efficiency. Section 2 presents the problem statement of this work. Even though this problem is common in many kinds of process industries, we will focus on a case study of an offshore compressor fleet of an oil and gas company, which is introduced in Section 3. A short overview of the mathematical formulation of the scheduling approach is given in Section 4 . The results of the maintenance scheduling are illustrated and discussed within 
the different subcases in Section 5. The conclusion gives an overview of the findings of this work and shows possible directions for future work.

\section{Problem Statement and Case Study}

The problem considered in this paper investigates how often online and offline washing in the context of other maintenance types should be conducted to optimally countermeasure deterioration effects and efficiency loss in compressors due to fouling. Furthermore, the actual benefit of washing can not always be determined beforehand. This information is important for the decision-making process; if a washing system should be installed on existing assets or not.

The case study that is used is inspired by a real fleet of compressors. In order to assess the performance of the formulation, the case study is analyzed with varying grid coarseness and time horizon length. For the length of the scheduling horizon a duration of 1-3 months was chosen, as this resembles the need for ahead-scheduling of maintenance workers and the ordering of specific resources such as spare parts or other supplies in most companies.

Table 1: Notation of the scheduling formulation

\begin{tabular}{llll}
\hline Index/Set & Description & $\begin{array}{l}\text { Continuous } \\
\text { Variable }\end{array}$ & Description \\
\hline$i$ & Task & $C_{r_{c}, t}$ & Enumerator \\
$j$ & Unit & $G_{r_{c}, t}$ & Goodness of unit \\
$t$ & Time Interval & $K$ & Profit/Cost term \\
$R$ & Resource & $\mu_{i, t}$ & Discrete interaction \\
$R_{c}$ & Resource (asset) & Binary Variable & Description \\
\cline { 2 - 3 }$T_{S}$ & Time (Scheduling) & $N_{i, t}$ & Maintenance task i starts at t \\
$I$ & Maintenance tasks & Parameters & Description \\
\cline { 2 - 3 } Superscript & Description & $D_{r_{c}}$ & Degradation factor \\
NonRecov & Non-recoverable & Superscript & Description \\
\cline { 3 - 4 } RInsp & degradation & $R O n W$ & $\begin{array}{l}\text { Recoverable degradation } \\
\text { (Online Washing) }\end{array}$ \\
Min & Recoverable degradation & ROffW & Recoverable degradation \\
(Inspection) & Minimum & Max & Maximum Washing) \\
\hline
\end{tabular}

\section{Mathematical Modeling}

The scheduling model is designed as a discrete-time model. While the exact starting times of maintenance actions depend on other external factors such as weather conditions or the changeover between two shifts, it is not important to work with a continuous-time model. A generic approach based on the Resource-Task Network (RTN) framework (Pantelides, 1994) is applied. The notation of the model is shown in Table 1.

The central element of the scheduling approach is the inherent goodness of every asset. This goodness is affected by degradation and therefore the efficiency decreases over time. 
As depicted in Figure 1 the efficiency of the compressor is decreasing over time. There are different terms for the various types of degradation and there are 5 distinctive maintenance modes that have particular effects. Different counters for each type of degradation are used to calculate the time passed since the last maintenance has taken place. Every conducted maintenance resets one or multiple counters, which are used to model the degradation in a linear fashion. The goodness degradation is defined as follows:

$$
\begin{aligned}
G_{r_{c}, t}= & G_{r_{c}}^{\text {initial }}-D_{r_{c}}^{\text {NonRecov }}\left(C_{r_{c}, t}^{G N}-1\right)-D_{r_{c}}^{R I n s p}\left(C_{r_{c}, t}^{R I n s p}-1\right) \\
& -D_{r_{c}}^{R O f f W}\left(C_{r_{c}, t}^{R O f W}-1\right)-D_{r_{c}}^{R O n W}\left(C_{r_{c}, t}^{R O n W}-1\right), \forall r_{c} \in R_{c}, t \in T_{S}
\end{aligned}
$$

The enumerators are each formulated in a comparable way. As an example, the enumerator $C_{r_{c}, t}^{R O n W}$ for counting the time intervals since the last online washing is introduced:

$$
\begin{aligned}
& C_{r_{c}, t}^{R O n W} \geq C_{r_{c}, t-1}^{R O n W}+1-M \sum_{i_{r_{c}}}^{I_{O n W}} N_{i, t}, \forall r_{c} \in R_{c}, t \in T_{S} \\
& C_{r_{c}, t}^{R O n W} \leq C_{r_{c}, t-1}^{R O n W}+1, \forall r_{c} \in R_{c}, t \in T_{S} \\
& C_{r_{c}, t}^{R O n W} \leq 1+M\left(1-\sum_{i_{r_{c}}}^{I_{O n W}} N_{i, t}\right), \forall r_{c} \in R_{c}, t \in T_{S} \\
& C_{r_{c}, t}^{R O n W} \geq 1, \forall r_{c} \in R_{c}, t \in T_{S}
\end{aligned}
$$

The enumerator for the Remaining Useful Life of the assets works in a similar fashion. However, it is reset by a short-term maintenance $I_{S M}$ that does not restore the goodness but fixes operational problems with the asset. The formulation is not shown in this paper. In a real-world application a continuous input from a Condition Monitoring System would be required.

The resource balance describes describes that every active maintenance task $N_{i, t}$ is consuming a specific amount of resources $\mu_{i}, t$ from the initial amount of resources $R_{0}$, including the compressors and the maintenance personnel. When a task is concluded, these resources are released again for the following time interval.

$$
R_{r, t}=R_{\text {initial }}-\sum_{i}^{I} N_{i, t} \mu_{i, t}, \forall r \in R, t \in T_{P}
$$

The target of the optimization is to maximize the profit, calculated by the production profit from each compressor, multiplied by the goodness of the compressor in each time interval minus the cost for maintenance.

$$
\max z=K_{\text {profit }} \sum_{\text {plat }}^{r_{c}} G_{r_{c}, t}-K_{i} \sum_{i} \sum_{t}^{T_{S}} N_{i, t}
$$


Table 2: Results of the washing scheduling for a fleet size of 10 compressors. (Legend: H - Time Horizon, G - Grid Size, Off - Offline Washing, On - Online Washing)

\begin{tabular}{|c|c|c|c|c|c|c|}
\hline \multirow[t]{2}{*}{ Case } & \multicolumn{3}{|c|}{ H: 1 month, G: 4 hours } & \multicolumn{3}{|c|}{ H: 2 month, G: 4 hours } \\
\hline & On & Off & On+Off & On & Off & On+Off \\
\hline Improvement $^{1}$ & $0.97 \%$ & $0.13 \%$ & $1.07 \%$ & $2.06 \%$ & $1.50 \%$ & $3.30 \%$ \\
\hline MIP-Gap & $0.72 \%$ & $0.46 \%$ & $0.61 \%$ & $7.03 \%$ & $4.45 \%$ & $2.24 \%$ \\
\hline \multirow[t]{3}{*}{ CPU-s ${ }^{2}$} & 1800 & 1800 & 1800 & 1800 & 1800 & 1800 \\
\hline & \multicolumn{3}{|c|}{ H: 1 month, G: 8 hours } & \multicolumn{3}{|c|}{ H: 2 months, G: 8 hours } \\
\hline & On & Off & On+Off & On & Off & On+Off \\
\hline Improvement $^{1}$ & $1.29 \%$ & $0.38 \%$ & $1.28 \%$ & $1.88 \%$ & $0.84 \%$ & $2.71 \%$ \\
\hline MIP-Gap & $0.26 \%$ & $0.01 \%$ & $0.28 \%$ & $3.42 \%$ & $2.91 \%$ & $1.19 \%$ \\
\hline CPU-s ${ }^{2}$ & 1800 & 1411 & 1800 & 1800 & 1800 & 1800 \\
\hline
\end{tabular}

\section{Results and Discussion}

Four different cases with fixed fleet size, but varying time horizon and grid size were solved using GAMS 24.8.4/CPLEX 12.7.1.0. The calculations are carried out as follows: Each case has the information that two different maintenance actions (long overhaul and short maintenance, e.g. change of a broken seal) must be conducted within the time horizon. This information could come in a real-world implementation e.g. from a higher level planning system. Furthermore, a specific combination of washing actions (online, offline or combined) can be conducted. The optimization problem tries to optimize production profit by recovering degradation of the compressors at the right time.

The improvement is calculated by comparing the three different scenarios for each case against the base-scenario in which no washing is performed, just the basic maintenance such as long overhauls and short-term maintenance. The quotient of the objective function for a specific scenario and the base scenario indicate the improvement.

The results of four different cases with three scenarios each are shown in Table 2. While the specific values of the objective function represent the entire production profit within the time horizon (see Eq. 7), the actual values of the production profit are not insightful. As the size of the optimization problem is larger the longer the time horizon is and the finer the grid size is, the scheduling algorithm is terminated prematurely in all cases at 1800 seconds. Therefore, the result shows the minimum improvement, as further calculation might improve the objective function. More exhaustive calculations may be conducted in a follow-up study.

The results show that the algorithm is able to improve the operating profit. The improvement ranges between $0.4 \%$ and $3.3 \%$. Several trends can be observed: The longer the time horizon is, the more improvement can be observed. As the overall degradation (nonrecoverable plus all recoverable degradation) is set to $5 \%$ per year, this makes sense, as smaller decreases in the efficiency do not necessarily justify conduction of washing, while 
on a longer time horizon washing is more economically viable. With fixed computational time, the gap between the best bound of the problem and the integer solution is dependent on the time horizon and the grid size and is thus scaling with the problem size.

\section{Conclusion}

A novel maintenance scheduling approach was presented in this work. The model differs from other work, as it combines the information about the asset degradation via an enumerator-approach into the decision-making for several types of maintenance actions, especially washing actions.

Degradation is considered to be a combination of recoverable degradation, non-recoverable degradation and the decreasing remaining useful lifetime of an asset. Results show that the scheduling of the proposed compressor washing in addition to other maintenance types is able to improve the production profit by up to $3.3 \%$ compared to operations without the compressor washing. Next to the fact that the scheduling model can give help to optimize the operation of process networks, it can be used as a decision-support tool. By comparing the possible saving introduced through new maintenance types, e.g. washing, a maximum capital investment for the amortization within a specific timeframe can be calculated.

Future work will investigate a multi-time-scale model that integrates both long-term planning and short-term scheduling of maintenance.

\section{Acknowledgment}

Financial support is gratefully acknowledged from the Marie Skłodowska Curie Horizon 2020 EID-ITN project "PROcess NeTwork Optimization (PRONTO)", Grant agreement No 675215.

\section{References}

I. S. Diakunchak, 1992. Performance Deterioration in Industrial Gas Turbines. Journal of Engineering for Gas Turbines and Power 114 (2), 161.

W. C. Elrod, P. I. King, E. M. Poniatowski, 1990. Effects of Surface Roughness, Freestream Turbulence, and Incidence Angle on the Performance of a 2-D Compressor Cascade. Volume 1: Turbomachinery, V001T01A061.

G. Hovland, M. Antoine, 2006. Scheduling of gas turbine compressor washing. Intelligent Automation and Soft Computing 12 (1), 63-73.

S. A. Khalid, A. S. Khalsa, I. A. Waitz, C. S. Tan, E. M. Greitzer, N. A. Cumpsty, J. J. Adamczyk, F. E. Marble, 1999. Endwall Blockage in Axial Compressors. Journal of Turbomachinery 121 (3), 499-509.

R. Kurz, K. Brun, 2001. Degradation in Gas Turbine Systems. Journal of Engineering for Gas Turbines and Power 123 (1), 70.

R. Kurz, K. Brun, 2012. Fouling Mechanisms in Axial Compressors. Journal of Engineering for Gas Turbines and Power 134 (3), 032401-032401.

C. C. Pantelides, 1994. Unified framework for optimal process planning and scheduling. In: Proc. Second Conf. on Foundations of Computer Aided Operations. pp. 253-274.

D. Singh, W. Tabakoff, E. Mechanics, 1996. Simulation of Performance Deterioration in Eroded Compressors. ASME. Turbo Expo: Power for Land, Sea, and Air Volume 1:, 1-8.

J.-P. Stalder, 2001. Gas Turbine Compressor Washing State of the Art: Field Experiences. Journal of Engineering for Gas Turbines and Power 123 (2), 363.

D. P. Xenos, G. M. Kopanos, M. Cicciotti, N. F. Thornhill, 2016. Operational optimization of networks of compressors considering condition-based maintenance. Computers and Chemical Engineering 84, 117131 